Published in final edited form as:

Anal Chem. 2007 November 15; 79(22): 8774-8780.

\title{
A Photoresist with Low Fluorescence for Bioanalytical
}

\section{Applications}

\author{
Jeng-Hao Pai ${ }^{1}$, Yuli Wang ${ }^{2}$, Gina To'A Salazar ${ }^{3,5}$, Christopher E. Sims ${ }^{5}$, Mark Bachman ${ }^{2,4}$, \\ G.P. Li ${ }^{2,4}$, and Nancy L. Allbritton ${ }^{\star}, 1,3,5$ \\ 1 Department of Chemical Engineering and Materials Science, University of California, Irvine, California, \\ 92697 \\ 2Integrated Nanosystems Research Facility, University of California, Irvine, California, 92697 \\ 3Department of Biomedical Engineering, University of California, Irvine, California, 92697 \\ 4Department of Electrical Engineering and Computer Science, University of California, Irvine, California, \\ 92697 \\ 5Department of Chemistry, University of North Carolina, Chapel Hill, NC 27599
}

\section{Abstract}

The negative photoresist SU-8 has found widespread use as a material in the fabrication of microelectrical-mechanical systems (MEMS). While SU-8 has been utilized as a structural material for biological MEMS, a number of SU-8 properties limit its application in these bioanalytical devices. These attributes include its brittleness, nonspecific adsorption of biomolecules, and high fluorescence in the visible wavelengths. In addition, native SU-8 is a poor substrate for cellular adhesion. Photoresists composed of resins with epoxide side groups and photoacids were screened for their ability to serve as a low fluorescence photoresist with sufficient resolution to generate microstructures with dimensions of 5-10 $\mu \mathrm{m}$. The fluorescence of structures formed from 1002F photoresist (1002F resin combined with triarylsulfonium hexafluoroantimonate salts) was as much as 10 times less fluorescent than similar SU-8 microstructures. The absorbance of 1002F in the visible wavelengths was also substantially lower than that of SU-8. Microstructures or pallets with an aspect ratio as high as $4: 1$ could be formed permitting $1002 \mathrm{~F}$ to be used as a structural material in the fabrication of arrays of pallets for sorting adherent cells. Several different cell types were able to adhere to native $1002 \mathrm{~F}$ surfaces and the viability of these cells was excellent. As with SU-8, 1002F has a weak adhesion to glass, a favorable attribute when the pallet arrays are used to sort adherent cells. A threshold, laserpulse energy of $3.5 \mu \mathrm{J}$ was required to release individual $50-\mu \mathrm{m}, 1002 \mathrm{~F}$ pallets from an array. Relative to SU-8, 1002F photoresist offers substantial improvements as a substrate in bioanalytical devices and is likely to find widespread use in BioMEMS.

\section{Introduction}

Negative photoresists are composed of a resin, a photoactive compound, and a solvent. ${ }^{1,2}$ When illuminated with light, the resin is converted to an insoluble form typically by undergoing an increase in molecular weight or by formation of a new insoluble molecule. The increase in molecular weight is generally achieved by polymerization or cross-linking of the resin monomers. Insolubility can also be attained by initiation of a photochemical reaction to alter polarity or hydrophobicity. For polymerization and cross-linking reactions, the photoactive compound is generally a photoinitiator which forms free radicals or strong acids upon exposure

\footnotetext{
* Corresponding author: NLA: 919-966-2291, Email: nlallbri@unc.edu, Fax: 919-962-2388.
} 
to light. Chemically amplified negative photoresists take this process one step further. Light is used to generate a chemical catalyst initiating a cascade of events, thus amplifying the effects of the initial photon absorption. ${ }^{3,4}$ Chemically amplified resists possess high sensitivity to UV light and provide high resolution structures. These resists have found a multitude of uses, for example in image reversal, as a protective layer during etching, and in formation of high aspect ratio microstructures. 5

SU-8 photoresist was introduced in 1989 by IBM as an chemically amplified, negative photoresist. ${ }^{1,6}$ SU-8 photoresist is comprised of the SU-8 resin (bisphenol-A novolac epoxy resin, CAS 28906-96-9), the photoinitiator triarylsulfonium hexafluoroantimonate salts, and a solvent, typically $\gamma$-butyrolactone (GBL) or cyclopentanone. In addition to its use as a highcontrast negative photoresist, $\mathrm{SU}-8$ also possesses a number of properties that make it attractive as a structural material in microfabricated devices. SU-8 is biocompatible, rigid, thermally and chemically stable, and transparent to light above $360 \mathrm{~nm} \cdot{ }^{1,7-11} \mathrm{SU}-8$ has been employed as a structural component in microelectrodes, atomic force microscopy tips, electrophoresis microchips, and other applications. ${ }^{12-22}$ While SU-8 has many assets, it has significant weaknesses when used as a structural material for bioanalytical applications. SU-8 is brittle and fractures easily. ${ }^{23,24}$ Native SU-8 is hydrophobic and prone to nonspecific adsorption of bioanalytes. ${ }^{25-28}$ In addition, most biological cells will not attach to native SU-8. Potentially the greatest weakness of SU-8 though is its high fluorescence in the visible wavelengths. ${ }^{17}$ Since fluorescence is the preferred detection method for many bioassays, the very high background fluorescence generated by SU-8 has limited its utility as a substrate in devices for biological studies.

A successful application of SU-8 as a structural component in a bioanalytical device is its use to form arrays of pallets to separate adherent cells. ${ }^{29}$ The pallet-array system permits cells or colonies of cells to be sorted while they remain on their growth surface broadening available selection criteria for the cells/colonies. The high aspect ratio of SU-8 allows a wide range of pallet heights $(25-100 \mu \mathrm{m})$ and sizes $(20-500 \mu \mathrm{m})$ to be constructed. Due to the good mechanical strength of SU-8, pallets dislodged from a glass surface using the focused beam of a pulsed laser remain intact. ${ }^{30}$ The optical transparency of SU- 8 has permitted a number of cell selection strategies to be applied to cells cultured on the SU-8 pallet arrays. These assays include selections utilizing transmitted light microscopy and fluorescence microscopy, particularly in the red wavelengths. ${ }^{31,32}$ Fluorescence detection in the green is also possible, but at greatly reduced sensitivity due to SU-8's high background fluorescence at these wavelengths. A second weakness of the SU-8 pallets is that they must be coated with adsorbed layers or covalently attached molecules before most cells will attach and grow on the pallet surface. ${ }^{31}$ A negative photoresist with decreased fluorescence and enhanced cell adhesive properties would broaden the use of the pallet arrays to applications requiring highly sensitive fluorescence detection and to separation of a wider range of cell types.

In the current work, we surveyed a variety of resins and photoinitiators to develop a low fluorescence alternative to SU-8 photoresist. The fabrication process for the new $1002 \mathrm{~F}$ photoresist was optimized including the spin-coating parameters, soft-bake times and temperatures, UV illumination time, and post-exposure baking procedure. In addition, the emission spectra as well as the absorbance at varying wavelengths was measured and compared to that of SU-8 and poly(dimethylsiloxane) (PDMS). Furthermore, the fluorescence of the $1002 \mathrm{~F}$ photoresist was compared to that of SU-8 using fluorescence microscopy filters typically used to view cellular fluorescence. The aspect ratio for the formation of arrays of pallets was defined as well as the adhesion and viability of cells on native 1002F photoresist. The ability of the $1002 \mathrm{~F}$ pallet arrays to support virtual walls which are required to localize cells to the pallet tops was characterized. Since the ultimate goal for the new photoresist was as a structural material for pallet arrays for cell sorting, the energy required to release $1002 \mathrm{~F}$ pallets from an 
array using a focused, pulsed laser was measured. These studies demonstrate the utility of the new $1002 \mathrm{~F}$ photoresist for the fabrication of arrays of pallets for use in the pallet array system.

\section{Experimental Section}

\section{Materials}

UVI-6976 photoinitiator (triarylsulfonium hexafluoroantimonate salts in propylene carbonate) was purchased from Dow Chemical (Torrance, CA) and poly(dimethylsiloxane) (PDMS) (Sylgard 184 silicone elastomer kit) was purchased from Dow Corning (Midland, MI). SU-8 photoresist and SU-8 developer (1-methoxy-2-propyl acetate) were obtained from MicroChem Corp. (Newton, MA). EPON resin 1002F (phenol, 4,4'-(1-methylethylidene)bis-, polymer with 2,2'-[(1-methylethylidene) bis(4,1-phenyleneoxymethylene]bis[oxirane]) was obtained from Miller-Stephenson (Sylmar, CA). All other photoinitiators and resins were from Sigma-Aldrich (St. Louis, MO) as were $\gamma$-butyrolactone (GBL), L-glutamine and poly(D-lysine)hydrobromide (MW 70,000-150,000). (Heptadecafluoro-1,1,2,2-tetrahydrodecyl)trichlorosilane was from Gelest Inc. (Morrisville, PA). Dulbecco's Modified Eagle Medium (DMEM), fetal bovine serum (FBS), and penicillin/streptomycin were obtained from Invitrogen (Carlsbad, CA). Cell proliferation kit II (XTT) was obtained from MD Biosciences Inc. (St. Paul, MN). Silicone Orings (24 mm outer diameter) were purchased from McMaster-Carr (Los Angeles, CA). All other reagents were obtained from Fisher Scientific (Pittsburgh, PA).

\section{Fabrication of Films and Pallets from SU-8 or 1002F Photoresist}

$1002 \mathrm{~F}-50$ photoresist was made by dissolving EPON resin $1002 \mathrm{~F}$ and triarylsulfonium hexafluoroantimonate salts in GBL at a ratio of $61 \%$ 1002F resin: $6.1 \%$ photoinitiator: $32.9 \%$ solvent (weight percentage). 1002F-10 photoresist was made of the same components but at a ratio of $49 \% 1002 \mathrm{~F}$ resin: $4.9 \%$ photoinitiator: $46.1 \%$ solvent. SU-8 and $1002 \mathrm{~F}$ films and pallets were fabricated on pre-cleaned glass slides $\left(75 \times 25 \times 1 \mathrm{~mm}^{3}\right)$. Films and pallets were fabricated in a manner similar to that previously described. ${ }^{29-31}$ SU-8 films with different thickness (10, $25,50,75$, and $100 \mu \mathrm{m}$ ) were obtained by spin-coating SU-8 photoresist on glass slides following the protocol provided by MicroChem Corp. $1,33,34$

To fabricate films and pallets composed of 1002F, 1002F films of different thickness (10-100 $\mu \mathrm{m}$ ) were obtained by spin coating $1002 \mathrm{~F}-50$ or $1002 \mathrm{~F}-10$ resist on a glass slide in a two step process as shown in Table 1 . The initial spin was for $10 \mathrm{~s}$ followed by a second spin for $30 \mathrm{~s}$. The coated slides were then soft baked on a hotplate at $65^{\circ} \mathrm{C}$ as shown in Table 1 , followed by a second bake at $95^{\circ} \mathrm{C}$ for the time indicated in Table 1 to remove organic solvent. After baking, the slides were allowed to slowly cool to room temperature on the hotplate ( $30 \mathrm{~min})$. To prepare an array of pallets, the 1002F film was exposed to UV light through a photomask with the appropriate design features for the times indicated in Table 1 using a collimated UV source $\left(6 \mathrm{~mW} / \mathrm{cm}^{2}\right.$, Oriel, Newport Stratford, Inc., Stratford, CT). The post-exposure bake was performed on a hotplate at $65^{\circ} \mathrm{C}$ followed by $95^{\circ} \mathrm{C}$ with the time at each temperature optimized for the thickness (Table 1). After cooling to room temperature, the $1002 \mathrm{~F}$ samples were developed in SU-8 developer, rinsed with 2-propanol, and dried in a stream of nitrogen.

\section{Collection of Fluorescence Emission Spectra}

SU- 8 or $1002 \mathrm{~F}$ photoresist or resin was poured into a mold $(3.5 \mathrm{~mL}, 1 \times 1 \times 3.5 \mathrm{~cm})$ made out of PDMS. The mold with photoresist or resin was placed in a vacuum oven at $100^{\circ} \mathrm{C}$ for 5 days to completely remove organic solvent. The mold and contents were then slowly cooled to room temperature. Spectra were then recorded from the sample blocks. The blocks were excited at $485 \mathrm{~nm}$ with a $\pm 2 \mathrm{~nm}$ band pass and the fluorescence measured from 490 to $650 \mathrm{~nm}$ with a \pm 2 $\mathrm{nm}$ band pass using a SpectraMax M5 microplate reader (Molecular Devices Corporation, 
Sunnyvale, CA). Data points were taken at $10 \mathrm{~nm}$ intervals. Fluorescence emission from cured blocks of PDMS was measured in a similar manner.

\section{Qualitative Characterization of SU-8 Fluorescence with Standard Microscopy Filter Sets}

Patterned SU-8 lines (100- $\mu \mathrm{m}$ width, 500- $\mu \mathrm{m}$ inter-line distance) of different heights $(10,25$, $50,75,100 \mu \mathrm{m}$ ) were fabricated on glass slides. SU-8 lines were then examined using a Nikon Eclipse TE300 inverted fluorescent microscope equipped with three fluorescent filter sets: a fluorescein filter set (B-2A, Nikon Instruments, excitation filter 450-490 nm, dichroic $500 \mathrm{~nm}$ long pass, emission $520 \mathrm{~nm}$ long pass), a tetramethyl rhodamine filter set (G-2E, Nikon Instruments, excitation filter 528-553 nm dichroic $565 \mathrm{~nm}$ long pass, emission 590-650 nm), and a Cy5 filter set (41008, Chroma Technology, excitation filter 590-650 nm, dichroic 660 $\mathrm{nm}$ long pass, emission 665-740 nm). A microscope objective (Nikon Plan Fluor, 20X, N.A. 0.50 ) was used to collect the fluorescent light which was detected using a cooled CCD camera (Photometrix Cool Snap $f x$, Roper Scientific, Tucson, AZ) controlled by Metafluor software (Molecular Devices, Sunnyvale, CA). For fluorescence imaging of cells, the signal-to-noise ratios of cells cultured on pallet arrays were determined using standard image analysis software (ImageJ, NIH).

\section{Measurement of SU-8 and 1002F Absorption}

To measure the absorbance of SU-8 and 1002F photoresists, films $(250 \mu \mathrm{m})$ were fabricated on a glass slide. The light absorption of the samples was measured with a JASCO V-530 UVVis spectrophotometer (Jasco Inc, Easton, MD) using air as a blank.

\section{Measurement of Cell Metabolism}

Rat basophilic leukemic (RBL) or human glioblastoma (A172) cells were cultured in a $37^{\circ} \mathrm{C}$, $5 \% \mathrm{CO}_{2}$ incubator at a density of 50,000 cells $/ \mathrm{mL}(100 \mu \mathrm{L})$ in chambers with bases constructed of glass, SU-8, or 1002F. To measure cell metabolism, XTT assays were performed on the cells at varying time points as per the manufacturer's instructions (Roche Applied Science, Indianapolis, IN). Cells from four separate chambers were assayed at each time point. The XTT assay measures the ability of cells to metabolize XTT (sodium 3 '-[1(phenylaminocarbonyl)-3,4-tetrazolium]-bis (4-methoxy-6-nitro) benzene sulfonic acid hydrate) to a colored product. The presence of the formazon product was determined by measuring the absorbance at $480 \mathrm{~nm}$ (SpectraMax M5, Molecular Devices Corporation, Sunnyvale, CA).

\section{Laser-Based Pallet Release}

Release of pallets by a single pulse ( $5 \mathrm{~ns}, 532 \mathrm{~nm}$ ) from a focused Nd:YAG laser (ACL-1, New Wave Research, Fremont, CA) was performed as described previously. ${ }^{30}$ Curves of the probability of pallet release with respect to the energy of the laser pulse were also constructed as described previously. ${ }^{30}$ The threshold energy for pallet release (energy at which $50 \%$ of the pallets released) was determined by fitting these curves to a Gaussian error function.

\section{Results and Discussion}

\section{Identification of Candidate Photoresists}

SU-8 photoresist exhibits substantial fluorescence in the visible wavelengths when excited with blue light (Fig. 1). Both SU-8 resin and the photoinitiator, triarylsulfonium hexafluoroantimonate salts possess complex ring structures that may act a source of this fluorescence (See supplemental data). To determine if either the resin or photoinitiator was the dominant source of the fluorescence, the fluorescence of the resin alone was measured (Fig. 1). When excited at $480 \mathrm{~nm}, \mathrm{SU}-8$ resin was still fluorescent although 3 times less so than the 
photoresist at $540 \mathrm{~nm}$. For comparison SU-8 photoresist is 800 times more fluorescent than PDMS at the same wavelengths. SU-8 resin was also less fluorescent than SU-8 photoresist at other wavelengths. However both the SU-8 resin and photoinitiator (or its decomposition products) acted as sources of fluorescence in the photoresist.

To determine whether a photoresist with similar properties to SU-8 but with lower fluorescence could be developed, a panel of photoinitiators and resins were screened for their ability to form microstructures of low fluorescence (Table 2 in the Supplementary Information Section). The photoinitiators were selected to be representative of both ionic photoacid generators (diaryliodonium and triarylsulfonium salts) and nonionic photoacid generators $(\mathrm{N}$ hydroxyimide sulfonates and others). The resins selected were solids at room temperature and possessed cross-linkable epoxy groups. The tested resins were poly(ethylene-co-glycidyl methacrylate), poly(ethylene-co-methyl acrylate-co-glycidyl methacrylate), tetraphenylolethane glycidyl ether, poly[(phenyl glycidyl ether)-co-dicyclopentadiene], and EPON 1002F resin (bisphenol A/epichlorohydrin-based epoxy resin) in addition to the SU-8 resin. Mixtures of the different combinations of photoinitiators and resins were spin-coated onto a glass or quartz surface. After an initial soft bake to remove the solvent, the mixtures were illuminated with UV light through a test mask. After a post-exposure bake, the sample was developed. The samples were assessed for their ability to act as a photoresist in the fabrication of microstructures as well as for the intensity of their fluorescence in the visible wavelengths. Of the tested mixtures, only the $1002 \mathrm{~F}$ resin with triarylsulfonium hexafluoroantimonate salts yielded high quality microstructures with a lower fluorescence than that of the SU-8 resin (Fig. 1,2).

\section{Characterization of 1002F Resin with Triarylsulfonium Hexafluoroantimonate Salts (1002F Photoresist) for the Fabrication of Pallets}

To further characterize the ability of $1002 \mathrm{~F}$ photoresist to act as a substrate for microstructures, a variety of different sized square and circular pallets with heights and diameters of 5-300 $\mu \mathrm{m}$ and $\geq 5 \mu \mathrm{m}$, respectively were fabricated. For each height, the ratio of the resin to photoinitiator in the resin was optimized as well as each of the fabrication steps (Table 1). When fabricated using these conditions, the pallets possessed the appropriate shapes with crisp edges by light microscopy (Fig. 2A). Pallets as small as $5 \mu \mathrm{m}$ could be fabricated (Fig. 2D). To view the side walls of the pallets, the structures were imaged by scanning electron microscopy. The side walls appeared smooth by SEM (Fig. 2B). For these pallets, the aspect ratio (height:width) was determined by fabricating structures with theoretical aspect ratios from 1:1 to 5:1. Ratios of 4:1 (Fig. 2C) and lower were successfully fabricated, but structures of 5:1 were not successfully formed. For comparison the maximal aspect ratio for SU-8 pallets was 10:1 using similar fabrication conditions. The aspect ratio of the $1002 \mathrm{~F}$ photoresist, however, was sufficient for the fabrication of pallets of a wide range of sizes for cell sorting applications.

\section{Fluorescence of 1002F Photoresist and Resin}

Since 1002F photoresist did possess fluorescence, it was important to identify the source of the fluorescence. A block of $1002 \mathrm{~F}$ resin was illuminated at $485 \mathrm{~nm}$ and the fluorescence at longer wavelengths measured and compared to that of an identical sized block of $1002 \mathrm{~F}$ photoresist. In the visible wavelengths, the $1002 \mathrm{~F}$ resin by itself was significantly less fluorescent than the 1002F photoresist (Fig. 1). At an emission wavelength of $540 \mathrm{~nm}$ the photoresist was over 5 times more fluorescent than the resin. These results suggested that the photoinitiator, triarylsulfonium hexafluoroantimonate salts, or its breakdown products were responsible for the majority of the $1002 \mathrm{~F}$ photoresist fluorescence. To further decrease the fluorescence of structures composed of $1002 \mathrm{~F}$, new low fluorescence photoinitiators will be required. 
Sorting biological cells from a mixture of cells is frequently performed by labeling the cells with a fluorescent reagent and then selecting those cells with the appropriate level of fluorescence. Therefore, it is important to understand the fluorescence properties of the $1002 \mathrm{~F}$ pallets using excitation and emission wavelengths commonly employed in fluorescence microscopy. Pallets of varying thickness were fabricated from SU-8 or 1002F photoresist and their fluorescence measured using filter sets designed for fluorescein, tetramethyl rhodamine, and Cy5 (Fig. 3A-C). As expected thicker pallets of SU-8 or 1002F exhibited greater fluorescence than thinner pallets. For all pallet thicknesses and excitation/emission wavelengths, the SU-8 pallets were substantially more fluorescent than the 1002F pallets. When excited at blue wavelengths, SU-8 pallets $50 \mu \mathrm{m}$ or greater in thickness possessed over an order of magnitude more fluorescence than the 1002F pallets (Fig. 3A). As the excitation/ emission wavelengths increased, the absolute fluorescence of both SU-8 and 1002F decreased. For green excitation wavelengths, the $1002 \mathrm{~F}$ pallets were as much as 10 times less fluorescent than SU-8 (Fig. 3B). When illuminated with red light, the 1002F pallets were nearly nonfluorescent while the SU-8 pallets continued to display significant fluorescence (Fig. 3C). These data demonstrate that $1002 \mathrm{~F}$ is a superior substrate for pallet fabrication when fluorescence is used for detection.

\section{Absorbance of 1002F Photoresist}

Many bioapplications using photoresists as a structural material employ absorbance measurements for analyte detection. For this reason, films of SU-8 or 1002F photoresist (250 $\mu \mathrm{m}$ thick) were coated on a slide. The films were exposed to UV light (without a photomask) and baked as described in the methods section. The absorbance of the films was then measured. SU-8 and 1002F possessed high transmittance at long wavelengths $(500-600 \mathrm{~nm})$ (Fig. 3D). Between 400 and $500 \mathrm{~nm}$, the absorbance of SU-8 increased as the wavelength decreased whereas $1002 \mathrm{~F}$ remained nearly transparent. By $400 \mathrm{~nm}$, the absorbance of SU-8 was greater than twice that of $1002 \mathrm{~F}$. The absorbance of both photoresists increased rapidly as the wavelength decreased below $400 \mathrm{~nm}$; however, at $370 \mathrm{~nm}$ the absorbance of $1002 \mathrm{~F}$ remained half that of SU-8. By $360 \mathrm{~nm}$, both photoresists possessed an absorbance greater than 1 . Neither SU-8 nor 1002F are good candidates as structural elements for measurements of absorbance in the ultraviolet wavelengths. However for wavelengths greater than $400 \mathrm{~nm}, 1002 \mathrm{~F}$ has excellent potential and is superior to SU-8 as a structural material for applications utilizing absorbance as a detection method. Other potential applications suitable for $1002 \mathrm{~F}$ include the fabrication of miniaturized, transparent, optical elements such as lenses.

\section{Cell Growth on 1002F Films}

Since cells have difficulty attaching to bare SU-8, coatings must be covalently attached or adsorbed onto the SU-8 surface in order for cells to attach. To assess the ability 1002F photoresist to support cell adhesion, RBL and A172 cells were cultured on $1002 \mathrm{~F}$ films placed on glass. Within 2-3 hours, cells were observed to attach and begin spreading on the $1002 \mathrm{~F}$ surface (data not shown). Other tested cells (rat fibroblast (208F) and human cervical carcinoma (HeLa)) behaved similarly.

To assess their longer term health, RBL and A172 cells were cultured on glass, SU-8, or 1002F surfaces for varying times and then assayed for their ability to metabolize XTT. In this assay, metabolically active cells convert XTT, a yellow tetrazolium salt, to an orange formazon product. Similar results were obtained for both cell types on the three different surfaces suggesting that none of the materials were toxic to these cells (Fig. 4A,B). The cells cultured on SU-8 possessed slightly slower XTT metabolism than those on glass or 1002F. Most likely this was due to the poor adhesion of cells to the SU-8 surfaces until the SU- 8 became fully coated with adsorbed proteins from the tissue culture medium. These results suggest that many cell types will be able to adhere to and grow on surfaces composed of native $1002 \mathrm{~F}$. 


\section{Cell Growth on Arrays of 1002F Pallets}

For arrays of $1002 \mathrm{~F}$ pallets to serve as a suitable format for cell selection and sorting, cells must attach to and grow on the top surfaces of the pallets and not on the glass surface between the pallets. A successful strategy to direct cells to the top surface of SU-8 pallets is to coat the array with a hydrophobic silane. ${ }^{29,30}$ Upon immersion of the SU-8 pallet array in an aqueous medium, air is stably trapped between the pallets forming a virtual wall between the pallets. These virtual walls exclude cells from the region between the pallets forcing the cells to attach to the top surfaces of the pallets. To determine whether $1002 \mathrm{~F}$ pallets could support virtual walls, an array of $1002 \mathrm{~F}$ pallets was coated with a perfluoroalkylsilane layer ((heptadecafluoro-1,1,2,2-tetrahydrodecyl)trichlorosilane). ${ }^{29}$ The array was then coated with collagen and immersed in an aqueous solution. Air trapped between the pallets was readily visualized as was the air:water interface at the edge of the pallet array (data not shown). To insure that these air walls could be used to localize cells to the pallet top surfaces, HeLa cells were cultured on the 1002F pallet array with virtual walls. Of $109 \mathrm{HeLa}$ cells visualized, 100\% were located on the pallet top surface with $0 \%$ on the intervening glass surfaces (Fig. 5A). Similar results were obtained for a collagen-coated, SU-8 array with virtual walls and cultured HeLa cells (Fig. 5B).

To assess the viability of the cells cultured on the 1002F array, the cells were loaded with viability indicator, Oregon Green diacetate. Living cells metabolize the dye to its counterpart, Oregon Green, which exhibits a green fluorescence. All cells on the $1002 \mathrm{~F}$ array metabolized and retained the dye demonstrating that they were viable (Fig. 5C). For comparison, RBL cells loaded with Oregon Green on an SU-8 array are also shown (Fig. 5D). The most notable difference between the images of the SU- 8 and $1002 \mathrm{~F}$ arrays is the high background fluorescence of the SU-8 pallets compared to the nonfluorescent, $1002 \mathrm{~F}$ pallets. The signalto-noise ratio was 2.7 for cells on SU-8 pallets, and 8.2 for cells on $1002 \mathrm{~F}$ pallets. These results demonstrate that the $1002 \mathrm{~F}$ photoresist can be used to form arrays of cells at known locations and with low background fluorescence.

\section{Laser-based release of $1002 \mathrm{~F}$ pallets from arrays}

A key aspect of the SU-8 pallet arrays is that individual pallets can be released by a single focused pulse from a Nd:YAG laser $(5 \mathrm{~ns}, 532 \mathrm{~nm}, 2 \mu \mathrm{J})$. On-demand, single-pallet release permits the collection of pallets possessing a desired cell and, consequently, the separation of that cell from the mixture of cells on the array. Thus, for $1002 \mathrm{~F}$ to be successful as a substrate for a low-fluorescence pallet array, the $1002 \mathrm{~F}$ pallets must be readily releasable from the array using low pulse energies. To ascertain whether $1002 \mathrm{~F}$ pallets were releasable, single pallets (50 $\mu \mathrm{m}$ side and height) in an array were targeted for release. When the laser pulse energy was greater than $4 \mu \mathrm{J}, 100 \%(\mathrm{n}=50)$ of the $1002 \mathrm{~F}$ pallets were released by a single pulse focused at the interface of the glass: $1002 \mathrm{~F}$ interface. None of the adjacent, untargeted pallets were released. To determine the threshold energy for pallet release, the probability of releasing a pallet was measured at varying pulse energies. The threshold energy for $1002 \mathrm{~F}$ pallet release was $3.5 \mu \mathrm{J}$. For comparison the threshold energy for identical-sized SU-8 pallets was $2.0 \mu \mathrm{J}$. While the energy for $1002 \mathrm{~F}$ pallet release was greater than that for SU-8 pallets, successful release and collection of living cells on SU-8 pallets has been performed with laser release energies up to $10 \mu \mathrm{J} .{ }^{32}$ Thus, the $1002 \mathrm{~F}$ pallets should be suitable for sorting many different types of cells.

There are several possible reasons for the higher energies required to detach the $1002 \mathrm{~F}$ pallets from the underlying glass surface relative to that for the SU-8 pallets. $1002 \mathrm{~F}$ has only two epoxide groups per monomer unlike SU- 8 which possesses 8 epoxides per monomer. Thus, while the SU-8 polymer is extensively crossed linked, 1002F is a linear, uncrossed-linked polymer. As a result $1002 \mathrm{~F}$ is a softer, more flexible material than SU-8. This difference is also 
observed when films of $1002 \mathrm{~F}$ and SU-8 are fabricated (data not shown). The softer, more flexible 1002F may absorb more mechanical energy before the contact with the glass is disrupted. In addition, these features may also afford $1002 \mathrm{~F}$ with a greater number of molecular interactions with the glass surface permitting a tighter bonding than that possible for the rigid SU-8. Alternatively, the lower absorbance of $1002 \mathrm{~F}$ compared to that of SU-8 near $532 \mathrm{~nm}$ may result in the conversion of a smaller fraction of the laser light into mechanical release energy and subsequent pallet detachment.

\section{Conclusion}

The use of $1002 \mathrm{~F}$ resin combined with triarylsulfonium hexafluoroantimonate salts as a photoresist was characterized and its applicability to the fabrication of arrays of pallets demonstrated. The fluorescence of the new $1002 \mathrm{~F}$ photoresist was significantly less than that of SU-8 enabling improved fluorescence sensitivity when $1002 \mathrm{~F}$ was used as a substrate. Most of the residual fluorescence of $1002 \mathrm{~F}$ structures is likely due to the fluorescence of the triarylsulfonium hexafluoroantimonate salts (or their byproducts). Thus, further reductions in the fluorescence await the development of photoacid generators with decreased fluorescence in the visible wavelengths. The aspect ratio of structures fabricated from $1002 \mathrm{~F}$ resin was $4: 1$ under these conditions and exceeds that needed for the fabrication of the micropallet arrays for cell sorting. These preliminary results also suggest that $1002 \mathrm{~F}$ is biocompatible in that cells grown on native or coated $1002 \mathrm{~F}$ demonstrated excellent adhesion and metabolic behavior. Both the ability of the $1002 \mathrm{~F}$ pallet arrays to support virtual walls as well as the low laser energies needed to detach $1002 \mathrm{~F}$ pallets from a glass substrate suggest that $1002 \mathrm{~F}$ is an excellent pallet substrate for the pallet array system. Moreover, $1002 \mathrm{~F}$ is similar to SU-8 in chemical structure, therefore, $1002 \mathrm{~F}$ can also be considered for applications currently using SU-8, such as DNA or protein immobilization to improve the signal-to-noise for fluorescence measurements. In addition to bioanalytical applications, the properties of $1002 \mathrm{~F}$ will enable this photoresist to serve as an excellent substrate for a variety other BioMEMS, for example, cantilevers, fluidic channels, reaction chambers, fluid dispensers, and cell culture reservoirs.

\section{Supplementary Material}

Refer to Web version on PubMed Central for supplementary material.

\section{Acknowledgements}

This research was supported by grants from the NIH.

\section{References}

1. Shaw JM, Gelorme JD, LaBianca NC, Conley WE, Holmes SJ. IBM J Res Dev 1997;41:81-94.

2. Wilson CG, Trinque BC. J Photopolymer Sci Tech 2003;16:621-628.

3. Ito H. IBM J Res Dev 2000;44:119-130.

4. Ito H. SPIE 1 1999;3678:2-12.

5. Madou, Marc J. Fundamentals of Microfabrication : The Science of Miniaturization. 2nd. CRC Press; Boca Raton, FL: 2002.

6. Gelorme, Jeffrey D.; Cox, Robert J.; Gutierrez, Sergio AR. Photoresist Composition and Printed Circuit Boards and Packages Made Therewith. IBM U.S. Patent 4,882, 245. 1989.

7. Lorenz H, Despont M, Fahrni N, LaBianca N, Renaud P, Vettiger M. J Micromech Microeng 1997;7:121-124.

8. Kotzar G, Freas M, Abel P, Fleischman A, Roy S, Zorman C, Moran JM, Melzak J. Biomaterials 2002;23:2737-2750. [PubMed: 12059024]

9. Voskerician G, Shive MS, Shawgo RS, Von Recum H, Anderson JM, Cima MJ, Langer R. Biomaterials 2003;24:1959-1967. [PubMed: 12615486] 
10. Belanger MC, Marois Y. J Biomed Mater Res B 2001;58:467-477.

11. O'Brien J, Hughes PJ, Brunet M, O'Neill B, Alderman J, Lane B, O'Riordan A, O'Driscoll C. J Micromech Microeng 2001;11:353-358.

12. Liu J, Bian C, Han J, Chen S, Xia S. Sens Actuators B 2005;106:591-601.

13. Kim GM, Kim B, Liebau M, Huskens J, Reinhoudt DN, Brugger J. J Microelectromechanical Sys 2002;11:175-181.

14. Zhang J, Tan KL, Gong HQ. Polymer Testing 2001;20:693-701.

15. El-Ali J, Perch-Nielsen IR, Poulsen CR, Bang DD, Telleman P, Wolff A. Sens Actuators A 2004;110:3-10.

16. Wakamoto Y, Inoue I, Moriguchi H, Yasuda K. J Anal Chem 2001;371:276-281.

17. Marie R, Schmid S, Johansson A, Ejsing L, Nordstrom M, Hafliger D, Christensen CBV, Boisen A, Dufva M. Biosens Bioelec 2006;21:1327-1332.

18. Kastantin MJ, Li S, Gadre AP, Wu LQ, Bentley WE, Payne GF, Rubloff GW, Ghodssi R. Sens Materials 2003;15:295-301.

19. Tseng FG, Lin KH, Hsu HT, Chieng CC. Sens Actuators A 2004;111:107-117.

20. Carlier J, Arscott S, Thomy V, Camart JC, Cren-Olive C, Le Gac S. J Chromatogr A 2005;1071:213222. [PubMed: 15865196]

21. Bilenberg B, Nielsen T, Clausen B, Kristensen AM. J Micromech Microeng 2004;14:814-818.

22. Su Y, Lin L. J MEMS 2004;13:75-82.

23. Feng R, Farris RJ. J Micromech Microeng 2003;13:80-88.

24. Khoo HS, Liu K, Tseng F. J Micromech Microeng 2003;13:822-831.

25. Grayson ACR, Shawgo RS, Johnson AM, Flynn NT, Li Y, Cima MJ, Langer R. Proc IEEE 2004;92:621.

26. Nordstrom M, Marie R, Calleja M, Boisen A. J Micromech Microeng 2004;14:1614-1617.

27. Whitesides GM, Ostuni E, Shuichi T, Jiang X, Ingber DE. Annu Rev Biomed Eng 2001;3:335-373. [PubMed: 11447067]

28. Wang Y, Bachman M, Sims CE, Li GP, Allbritton NL. Langmuir 2006;22:2719-2725. [PubMed: 16519474]

29. Wang Y, Sims CE, Marc P, Bachman M, Li GP, Allbritton NL. Langmuir 2006;22:8257-8262. [PubMed: 16952271]

30. Salazar GT, Wang Y, Young G, Bachman M, Sims CE, Li GP, Allbritton NL. Anal Chem 2007;79:682-687. [PubMed: 17222037]

31. Wang Y, Young G, Bachman M, Sims CE, Li GP, Allbritton NL. Anal Chem 2007;79:2359-2366. [PubMed: 17288466]

32. Wang Y, Young G, Phillip CA, Pai JH, Bachman M, Li GP, Sims CE, Allbritton NL. Cytometry A. 200710.1002/cyto.a.20424Published Online: 8 Jun 2007

33. http://www.microchem.com/products/pdf/SU8_2-25.pdf

34. http://www.microchem.com/products/pdf/SU8_50-100.pdf 


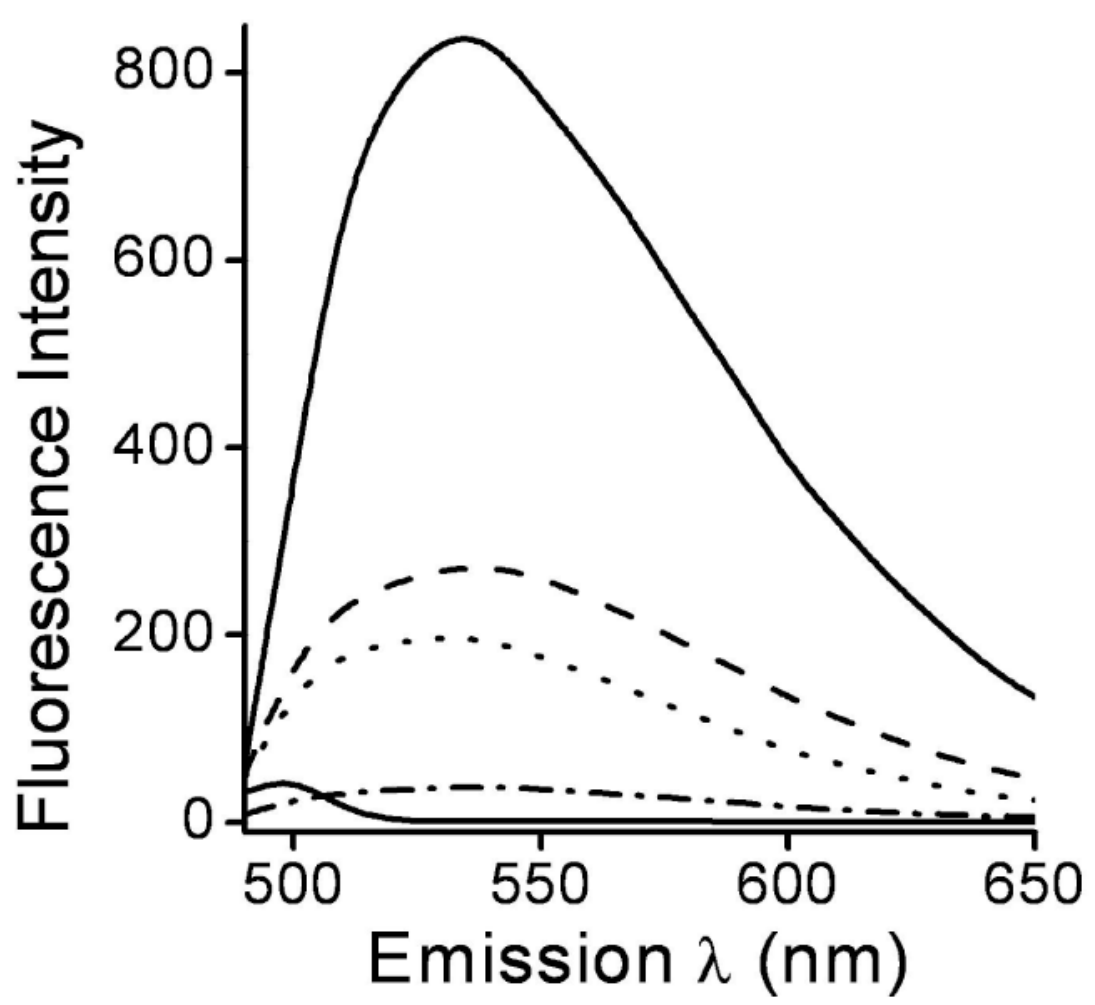

Figure 1.

Fluorescence of blocks of SU-8 and 1002F resin and photoresist. The blocks were excited at $485 \mathrm{~nm}$ with a $\pm 2 \mathrm{~nm}$ band pass and the fluorescence measured from 490 to $650 \mathrm{~nm}$ with a \pm 2 $\mathrm{nm}$ band pass. The upper solid and dashed lines are SU-8 photoresist and SU-8 resin, respectively. The dotted and dash-dotted line are $1002 \mathrm{~F}$ photoresist and $1002 \mathrm{~F}$ resin, respectively. The lower solid line is PDMS. For technical reasons, the photoresist blocks were not treated with UV exposure and post-exposure baking. 


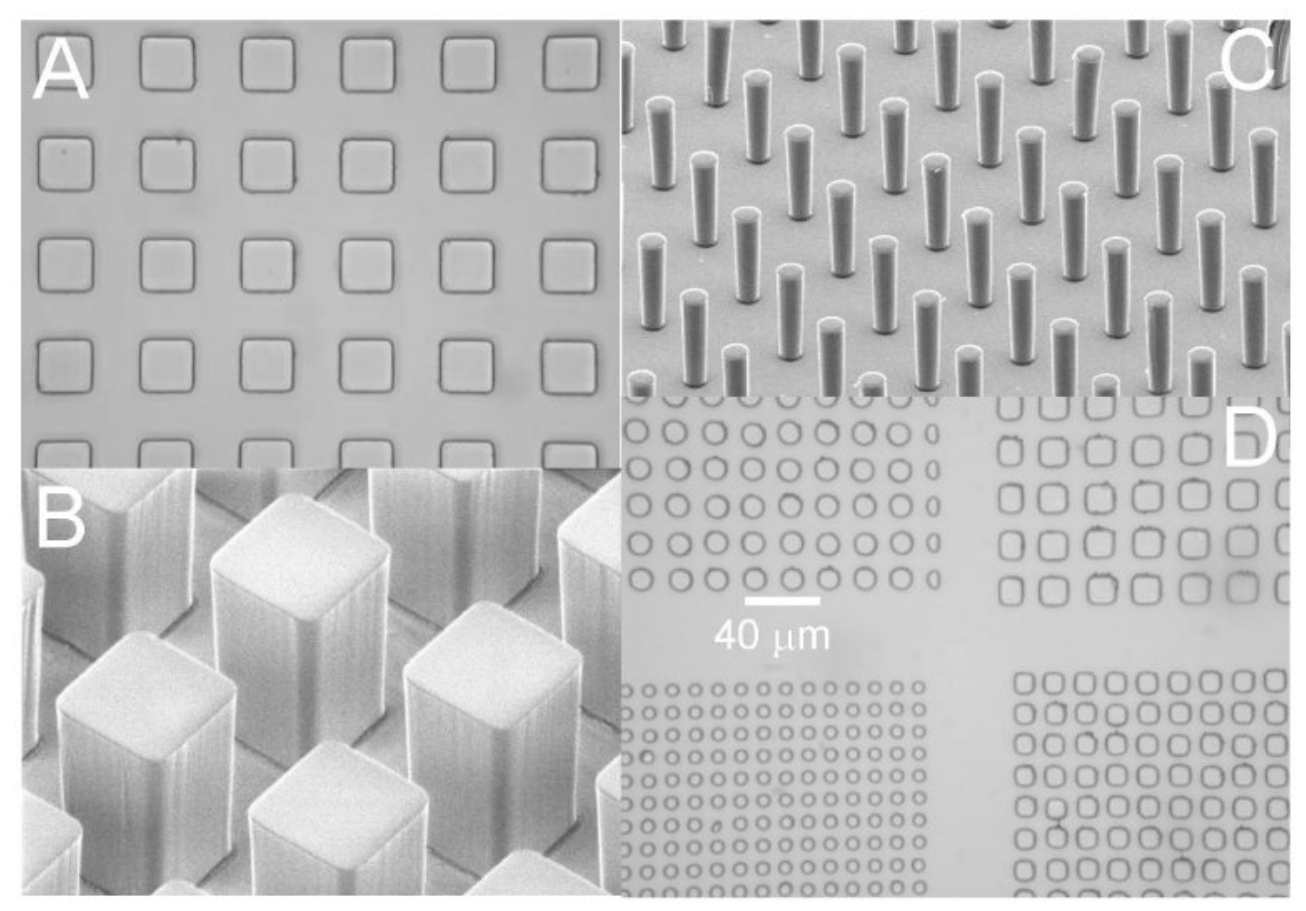

Figure 2.

Images of $1002 \mathrm{~F}$ pallets. (A) Transmitted light microscopy of an array of pallets. Each pallet was square in cross section with a $50 \mu \mathrm{m}$ side and $75 \mu \mathrm{m}$ height. The distance between each pallet was $50 \mu \mathrm{m}$. (B) SEM of the pallets shown in (A). (C) SEM of an array of pallets. Each pallet was circular in cross-section with a $25 \mu \mathrm{m}$ diameter and $100 \mu \mathrm{m}$ height. (D) Transmitted light microscopy of four pallet arrays fabricated on a single glass slide. The pallets on the left side of the image were fabricated from a mask with circular openings of diameter $10 \mu \mathrm{m}$ (top array) or $5 \mu \mathrm{m}$ (bottom array). The pallets on the right side of the image were square openings with a side of $10 \mu \mathrm{m}$ (top array) or $5 \mu \mathrm{m}$ (bottom array). The spacing between the 5 - $\mu \mathrm{m}$ and $10-\mu \mathrm{m}$ pallets was $5 \mu \mathrm{m}$ and $10 \mu \mathrm{m}$, respectively. 

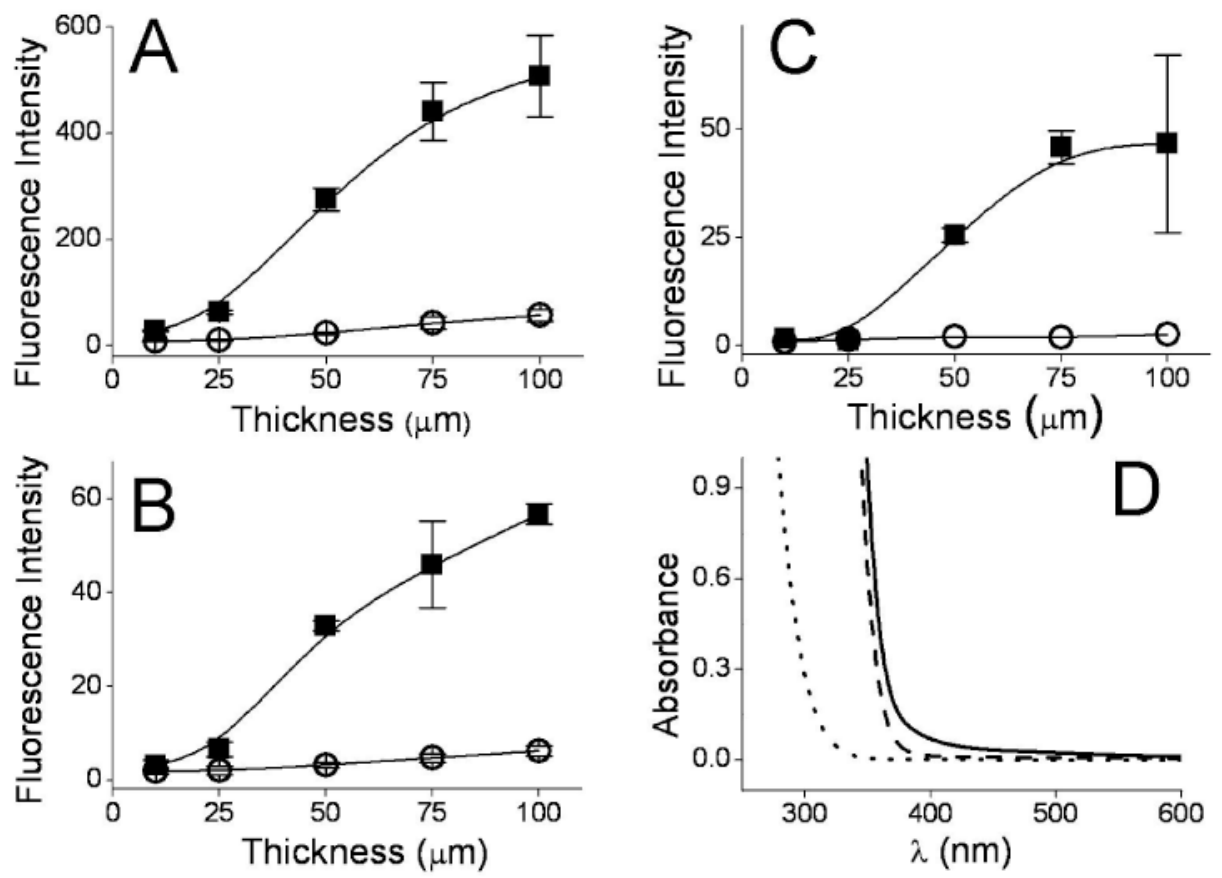

Figure 3.

Spectral properties of SU-8 and 1002F photoresists after UV exposure and post-exposure bake. (A) Fluorescence of SU-8 (solid squares) and 1002F (open circles) of lines (100 $\mu \mathrm{m}$ wide) of varying heights. The fluorescence was collected with a filter set designed for fluorescein (excitation filter $450-490 \mathrm{~nm}$, dichroic $500 \mathrm{~nm}$ long pass, emission $520 \mathrm{~nm}$ long pass). (B,C) Same as (A) but the fluorescence was collected using a filter set for tetramethyl rhodamine (excitation filter 528-553 nm dichroic $565 \mathrm{~nm}$ long pass, emission 590-650 nm) (B) or Cy5 (excitation filter 590-650 nm, dichroic $660 \mathrm{~nm}$ long pass, emission 665-740 nm) (C). The error bars represent the standard deviation of 5 measurements. (D) The absorbance of glass (dotted line), $1002 \mathrm{~F}$ photoresist (dashed line), and SU-8 photoresist (solid line) is shown at varying wavelengths. 

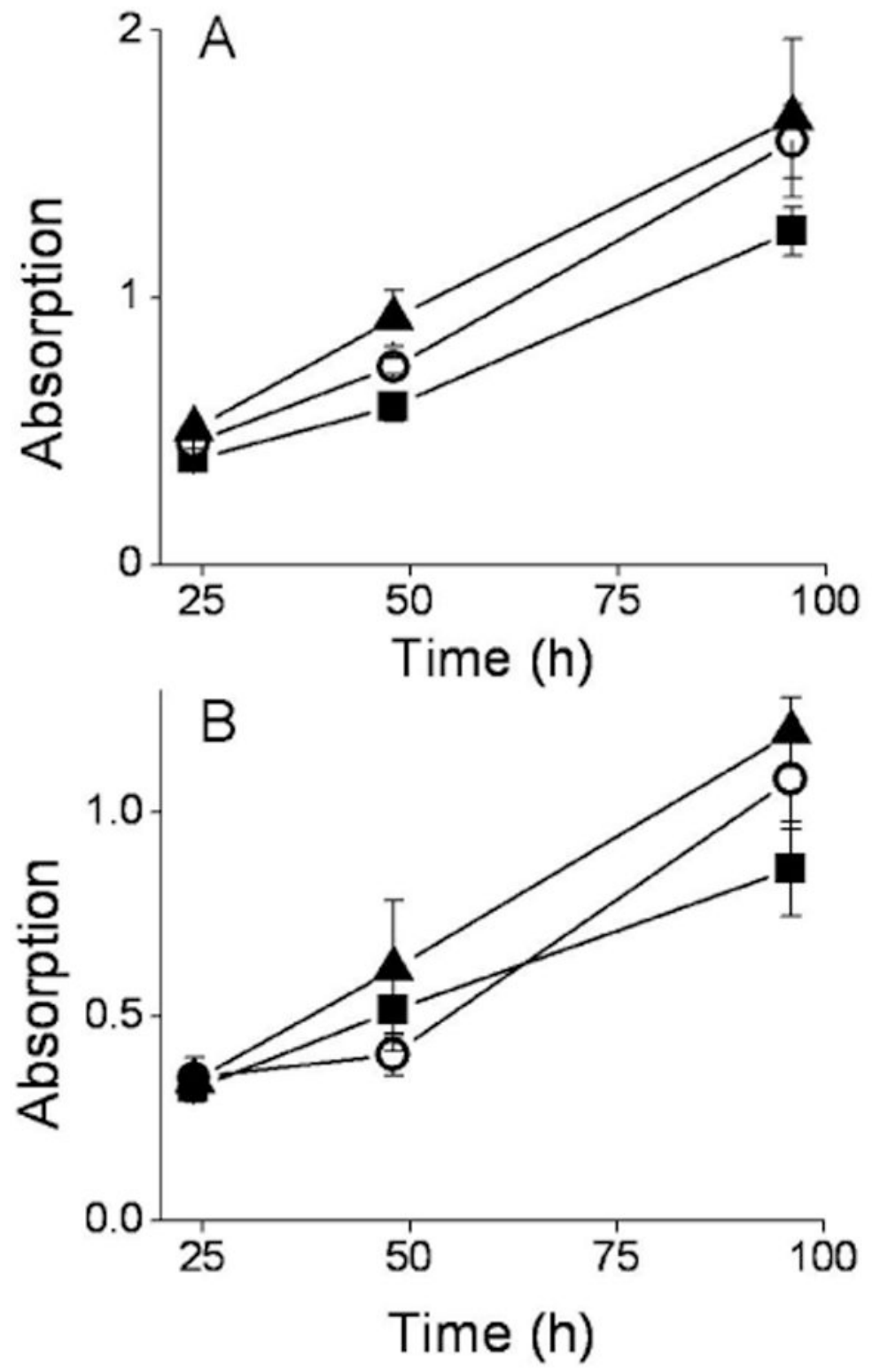

Figure 4.

Measurement of metabolism by XTT assay of cells grown on uncoated photoresists. RBL (A) or A172 (B) cells were cultured on glass (solid triangles), 1002F photoresists (open circles), or SU-8 photoresist (solid squares) for varying times. Shown on the "y" axis is the absorbance of the orange formazon product produced by metabolically active cells. The error bars represent the standard deviation of 4 measurements. 

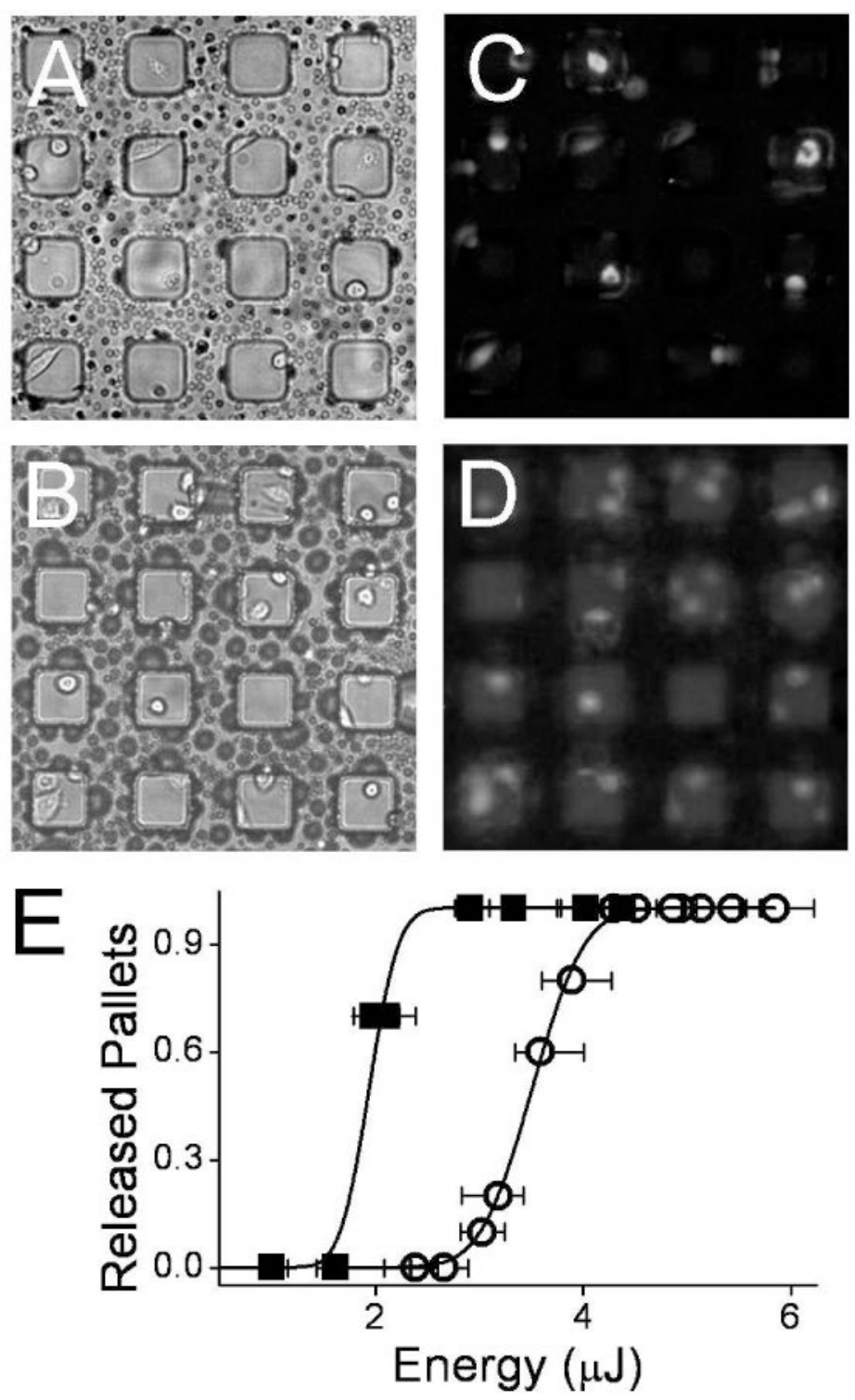

Figure 5.

Properties of SU-8 and 1002F pallets fabricated in arrays. (A,B) Transmitted light images of HeLa cells grown on 1002F (A) or SU-8 (B) pallets on arrays with virtual walls. (C,D)

Fluorescence images of HeLa cells loaded with Oregon Green on 1002F (C) or SU-8 (D) pallet arrays. (E) Measurement of the threshold energy for pallet release. Shown on the "y" axis is the fraction of pallets released at each energy tested. Ten pallets were released for each data point. The energy of each pulse sent from the laser was measured. The " $x$ " coordinate represents the average energy at a given laser setting and the error bars represent the standard deviation. The dimensions of the pallets in (A)-(E) were $50 \mu \mathrm{m}$ (side) and $100 \mu \mathrm{m}$ (height). The distance between each pallet was $50 \mu \mathrm{m}$. 


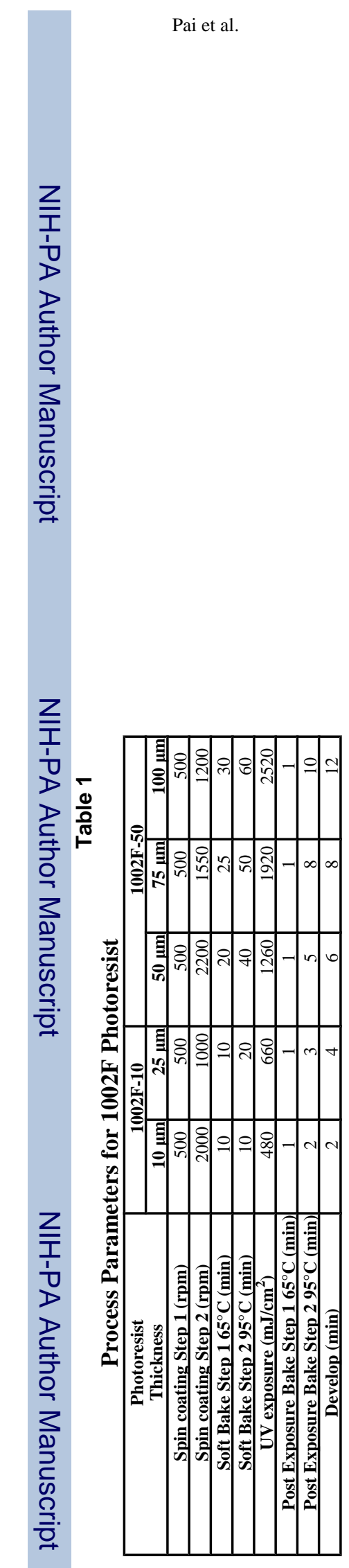

Anal Chem. Author manuscript; available in PMC 2008 June 22. 